\title{
Erratum to: Analysis of juvenile tuna movements as correlated random walk
}

\author{
Minoru Kadota $\cdot$ Shinsuke Torisawa $\cdot$ \\ Tsutomu Takagi $\cdot$ Kazuyoshi Komeyama \\ Hiromu Fukuda
}

Published online: 25 November 2011

(C) The Japanese Society of Fisheries Science 2011

Erratum to: Fish Sci (2011) 77:993-998

DOI 10.1007/s12562-011-0415-y

The original version of this article unfortunately contained a mistake. The author Hiromu Fukuda was not included.

The online version of the original article can be found under doi:10.1007/s12562-011-0415-y.

M. Kadota $(\bowtie) \cdot$ S. Torisawa $\cdot$ T. Takagi $\cdot$ H. Fukuda Department of Fisheries, Faculty of Agriculture,

Kinki University, 3327-204 Naka-machi,

Nara 631-8505, Japan

e-mail: kadota@cims.nyu.edu

K. Komeyama

The Education and Research Center for Marine Resources

and Environment, Faculty of Fisheries,

Kagoshima University, 4-50-20 Shimoarata,

Kagoshima 890-0056, Japan 